\title{
Diffraction of Phase Conjugate Material in a New HMD Architecture
}

\author{
Ricardo Martins and Jannick Rolland \\ School of Optics / CREOL \\ University of Central Florida, Orlando FL \\ ricky@odalab.ucf.edu \\ jannick@odalab.ucf.edu
}

\begin{abstract}
Conventional head-mounted displays (HMDs) consisting of a pair of miniature projection lenses, beam splitters, and miniature displays mounted on the helmet, as well as phase conjugate material placed strategically in the environment have been redesigned to integrate the phase-conjugate material into a complete see-through embodiment. Some initial efforts of demonstrating the concept was followed by an investigation of the diffraction effects versus image degradation caused by integrating the phase-conjugate material internally in the HMD. The key contribution of this paper lies in the conception, and assessment of a novel see-through HMD. Finally, the diffraction efficiency of the phase-conjugate material is evaluated, and the overall performance of the optics is assessed in both object space for the optical designer and visual space for possible users for this technology.
\end{abstract}

\section{INTRODUCTION}

$3 \mathrm{D}$ visualization devices, which have succeeded in penetrating real world markets, have evolved into three formats: standard monitors/shutter glasses, head-mounted displays (HMDs), and projection-based displays such as CAVEs. ${ }^{1}$ Each of the three common approaches currently imposes a significant increase in cost. In addition, monitors with shutter glasses are limited in capability, and CAVES are prohibitive in cost and limited to fully support only one user at a time without perceptual distortions. HMDs currently provide a fine balance of affordability and unique capabilities such as creating mobile and secure displays, ${ }^{2}$ spanning the virtual environments continuum first proposed by Milgram (1994), ${ }^{3}$ and enabling teleportal capability with face-to-face interaction. ${ }^{4}$

Most future display technologies will be linked to the telecommunication networks. Mobile and distributed systems are driven by concrete real world applications testable in real environments. The overall thrust of the research is to develop HMD technologies that support outdoor helmet mounted displays specifically aimed at mobile augmented reality navigation and information systems. This effort led to the conceptual novel design of a see-through HMD that will provide a solution for an improved outdoor virtual environment.

A recent novel type of HMD is the head-mounted projection display (HMPD), which may be thought as a miniature projector mounted on the head with a phase conjugate material placed strategically in the environment. In Fig. 1. the display presented in this paper builds on the HMPD concept, however the novelty lies in the integration of the phase conjugate material within the HMD. Such display is also light weight but can be used outdoors. ${ }^{5}$ The weight of the optics is less than $8 \mathrm{~g}$ per eye in the current conception. This technology developed lies beyond the boundaries of any conventional HMDs and projection-based displays because it opens the door from an indoor environment tethered to the phase conjugate material placed in the environment to a mobile system with the capability to be outdoors. Such configuration allows 3D visualization capability with a large FOV (i.e. $30^{\circ}<\mathrm{FOV}<90^{\circ}$ diagonal), lightweight optics (i.e. 8 g per eye) and low distortion (i.e. $<1.5 \%$ at the edge of the FOV). Distortion may easily be constrained at the expense of other field aberrations to be less than one percent depending on the targeted 
application because of the pupil location within the optics by design. A potential drawback of the new HMD compared to the original HMPD is the loss of some of the natural occlusion cues that might be desired in targeted applications.

In this paper the conceptual design of the HMD is presented in Section 2. In Section 3, the optical design is detailed. Finally, Section 4 presents an analysis of diffraction blur and experimental validation.
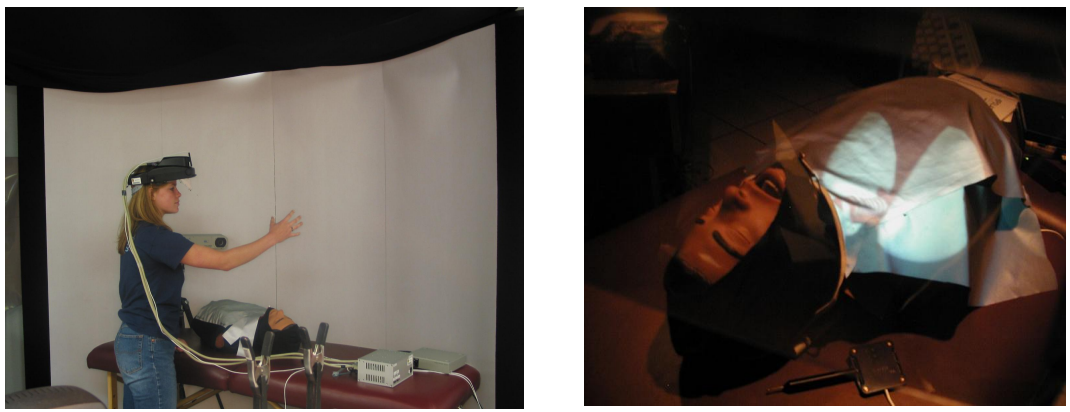

Figure 1 Current HMPD

\section{CONCEPT OF A NEW SEE-THROUGH HEAD-MOUNTED DISPLAYS (HMD)}

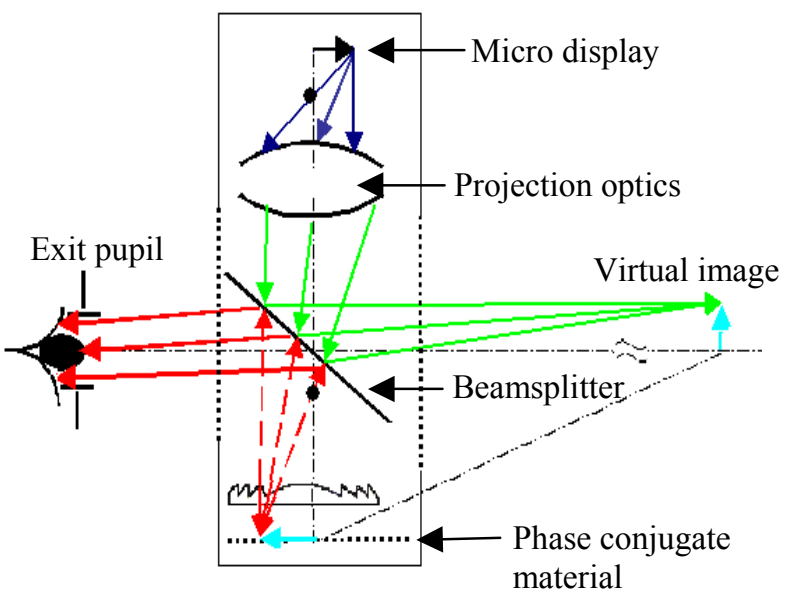

Figure 2 Conceptual design
The conceptual design for the see-through HMD was achieved and patented in the Optical Diagnostics and Application Laboratory (ODA Lab) and the actual design was finalized under the Synthetic Natural Environment program of the US Army. This design shown in Fig. 2 has incorporated projection optics and phase conjugate material within the HMD, thus eliminating the requisite use of an external phase conjugate material. ${ }^{6}$ A key component of this design is not only the integration of the phase conjugate material and projection optics but also the use of a lens located near the material that facilitates the operability of this technology. Because of its stand alone capability, this display extends the use of projection head mounted displays to clinical guided surgery, medical simulation training, and outdoor augmented see-through virtual environment for military training and wearable computers.

In a first design layout, Fig. 3 provides an illustration of how the see-through HMD can be worn on a user's head. From this design it is not sufficient to solely place the phase conjugate material in close proximity to the user's head because of the vast amount of diffraction blur (i.e. approximately 9.7 arcmin) caused if we discard the use of an additional optical element. Therefore, the use of an optical element was implemented in order to image the phase conjugate material at or approximately near the virtual image plane. 


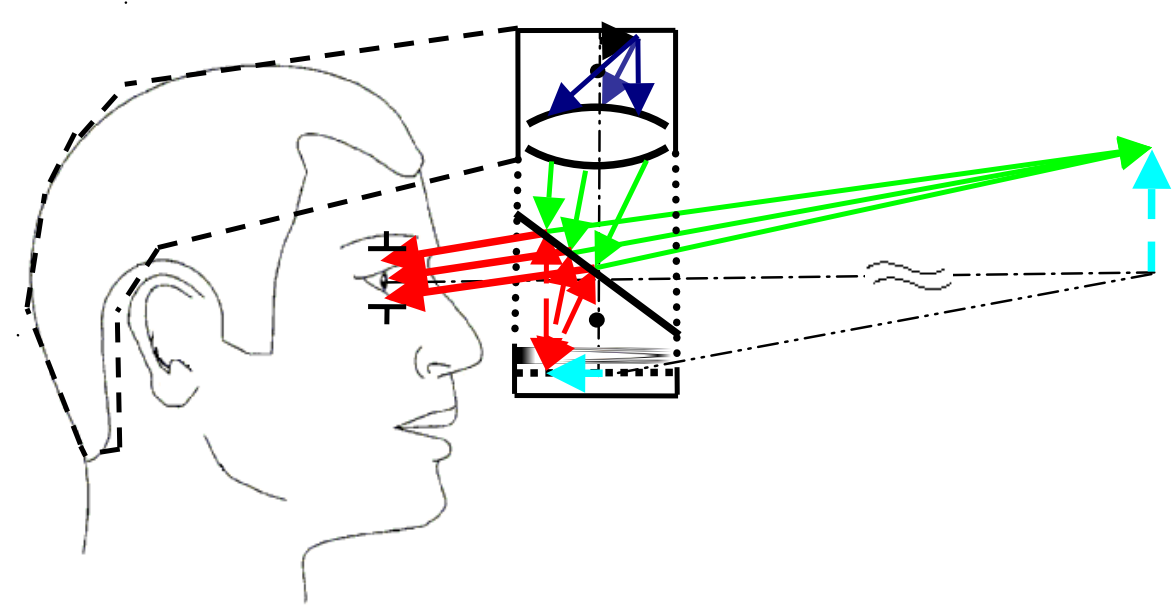

Figure 3 Design Layout

\section{OPTICAL LENS DESIGN}

\begin{tabular}{|l|l|}
\hline \multicolumn{1}{|c|}{ Parameter } & \multicolumn{1}{c|}{ Specification } \\
\hline Object: Color OLED & $0.6^{\prime \prime}$ inch in diagonal \\
\hline a. Size & Rectangle, $9 \mathrm{~mm} \times 12 \mathrm{~mm}$ \\
\hline b. Active display area & $\begin{array}{l}800 \mathrm{x} 600 \text { pixels } \\
1.3 \text { arcmin }\end{array}$ \\
\hline c. Resolution & \\
\hline Lens: & Projection lens \\
\hline a. Type & $19.5 \mathrm{~mm}$ \\
\hline b. Effective focal length & $12 \mathrm{~mm}$ \\
\hline c. Exit pupil diameter & $25.7 \mathrm{~mm}$ \\
\hline d. Overall length & 1 \\
\hline e. No. of diffractive surface & \\
\hline Other Parameters: & $486-656 \mathrm{~nm}$ \\
\hline a. Wavelength range & $42.0^{\circ}$ in diagonal \\
\hline b. FOV & $<1.5 \%$ over entire FOV \\
\hline c. Distortion & \\
\hline
\end{tabular}

The projection optics of the HMPD is composed of a binocular system, which consists of two identical optical lenses. The difference in the design of a projection lens for the HMD from other common projection optics is the requirement for lightweight and compactness. In the optical design of the HMD, we employed a combination of plastic, glass, and diffractive optics in order to reach lightweight and compactness. The miniature display selected was based on illumination requirements and was a 0.6" Organic Light Emitting Diode (OLED), manufactured by eMagin Corp., with 800x600 pixels and a $50-\mu \mathrm{m}$ pixel size. Given the miniature display, wide field-ofview (FOV) and high resolution is always two contradictory but desirable requirements. $^{7}$ Besides the consideration of resolution,

Table 1 Specification of projection optics there are two aspects of limitation on the targeted FOV. One aspect is that a flat beam splitter imposes a maximum FOV of $90^{\circ}$; the other aspect is the significant retro-reflectivity drop-off of commercially available phase conjugate materials beyond $\pm 35^{\circ}$ of incidence, which imposes an upper limit of $70^{\circ}$ on FOV for a flat retro-reflective screen. ${ }^{8}$ Table 1 summarizes the overall design specifications of the $42^{\circ}$ optics for the see-through head-mounted display. 
The starting point of this design is a patented 4-element lens shown in Fig. 4. ${ }^{9}$ The HMPD built with this optics is also shown in Fig. 5. In this design, in order to achieve lightweight, both the aspheric and the DOE lenses are made of plastic. The overall weight of the lens system is about 8 grams per eye. Fig. 6 shows the layout of the optical system that will be integrated in the new HMD. The purpose of employing a DOE is to correct the secondary spectrum and residual spherical aberrations for apochromatic imaging, in place of using high-index lanthanum crown glasses. $^{9-12}$

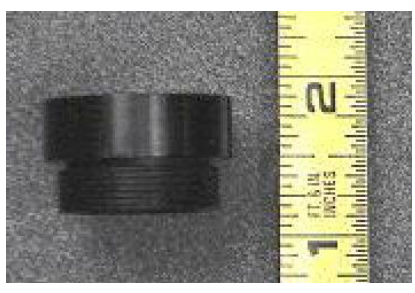

Figure 4 Lens assembly

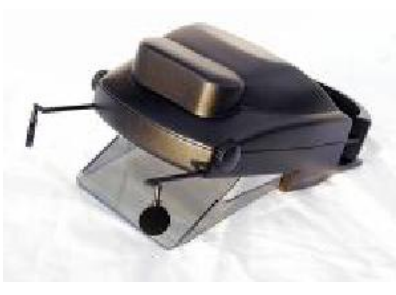

Figure 5 Head-mounted projection display

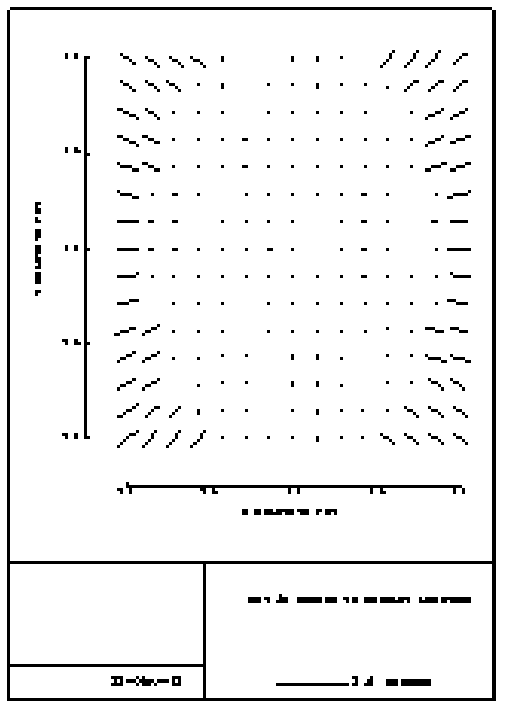

Figure 8 Astigmatism in arcmin
Fig. 7 shows the polychromatic diffraction modulation transfer function (MTF) for the full 12-mm pupil, which is presented across the five representative field angles. The target OLED display (see Table 1) has a spatial frequency of $24 \mathrm{lp} / \mathrm{mm}$ given a $50-\mu \mathrm{m}$ pixel size. We note that the modulation ratio of the design at $24 \mathrm{lp} / \mathrm{mm}$ is approximately $60 \%$ across the FOV. Therefore, we can scale the FOV without reducing the MTF below a design criterion of $20 \%$ at $24 \mathrm{lp} / \mathrm{m}$, but we must consider that the performance is currently limited by the miniature display size. In the HMD optics, the main aberrations to control were astigmatism and field curvature, which means a perfect point on the miniature display can either be displayed in visual space as a blurred spot or as an elongated line due to these aberrations. ${ }^{12}$ Therefore, to minimize any residual aberrations in visual space a field lens near the miniature display was placed to compensate and correct these effects. An analysis of the optical design shown in Fig. 8 illustrates the amount of astigmatism in visual space across the FOV in term of the visual measurement in arc minutes, and the direction of the lines show the direction along which a perfect point would be elongated in visual space. The result shows that across the FOV, the accommodation shift and astigmatism are less than 1.2 arc minutes. After designing the projection optical system, a future endeavor will be to fabricate and assemble the complete see-through HMD as shown in Fig. 3. 


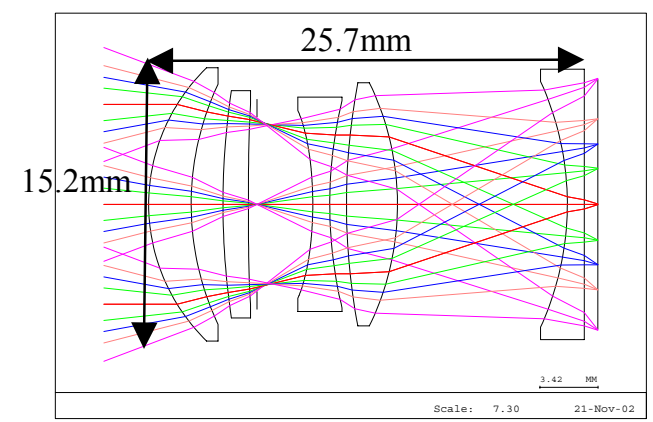

Figure 6 Projection optics

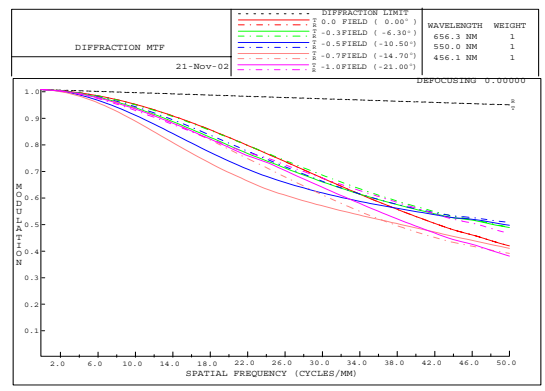

Figure 7 MTF curves

\section{EXPERIMENTAL RESULTS OF PHASE CONJUGATE MATERIAL}

Placing the phase conjugate material internally in the HMD we investigated two commercially available types of retro-reflective material: micro-beads and micro-corner-cube arrays. The micro-bead arrays operate on specular reflection, whereas the micro-corner-cube arrays utilize total internal reflection (TIR). At the current status of commercially available retro-reflective materials, manufacturers have yet to optimize the material for imaging conditions. Instead the material is currently specific for traffic control and other safety applications. For an ideal case of a perfect phase conjugation the incoming rays emitted by the micro display should be retroreflected with respect to the incident light without any deviation. Furthermore, the retro-reflected rays are not returned individually, instead a cone of diffracted light is returned producing an amount of image degradation. The amount of light in the observation plane depends on the microstructure and the retro-reflective properties of the materials employed. ${ }^{8}$ We can define the amount of light retro-reflected from the phase conjugate material to the user's eye by basing the analysis on the diffraction efficiency of the microstructure geometry. This approach yields accurate results, providing that first the microstructure is large compared to the wavelength, and the diffracted field is observed far from the phase conjugate material; based on these conditions we can treat the diffraction of light as a scalar phenomenon.

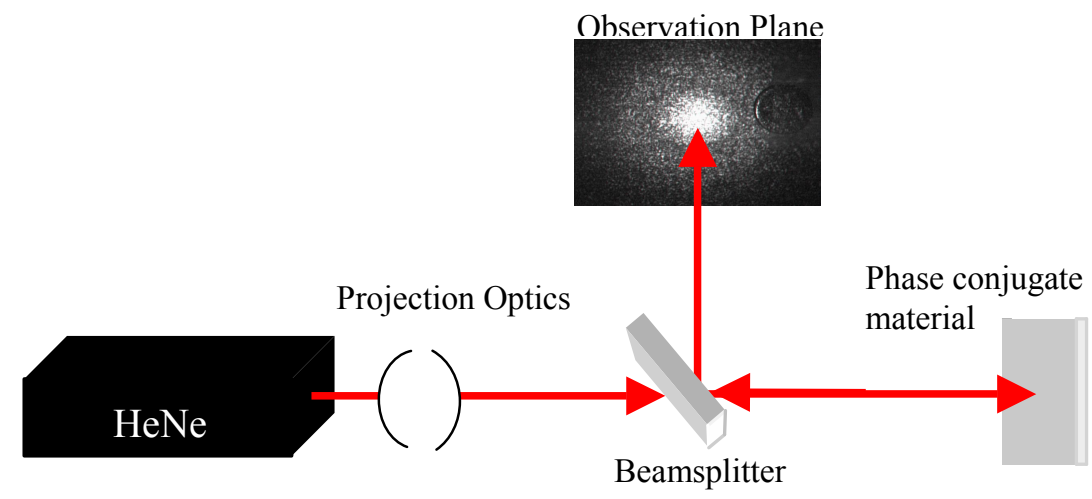

Figure 9 Experimental setup to investigate diffraction of the microstructure

A theoretical formulation is provided to verify some experimental data from the setup shown in Fig. 9. To characterize the imaging with retro-reflective materials, we consider the point-spread function (PSF) given by the modulus square of the complex amplitude in the image plane $\left|U\left(x_{2}, y_{2}, z\right)\right|^{2}$. We consider for simplicity an imaging scheme in the far field condition where $z>>k / 2\left(x_{1}^{2}+y_{1}^{2}\right)$, and where we express the intensity $I\left(x_{2}, y_{2}, z\right)$ at the image plane, located away 
from the microstructure in the general case, caused by diffraction of the microstructure geometry $A\left(x_{1}, y_{1}, 0\right){ }^{13}$

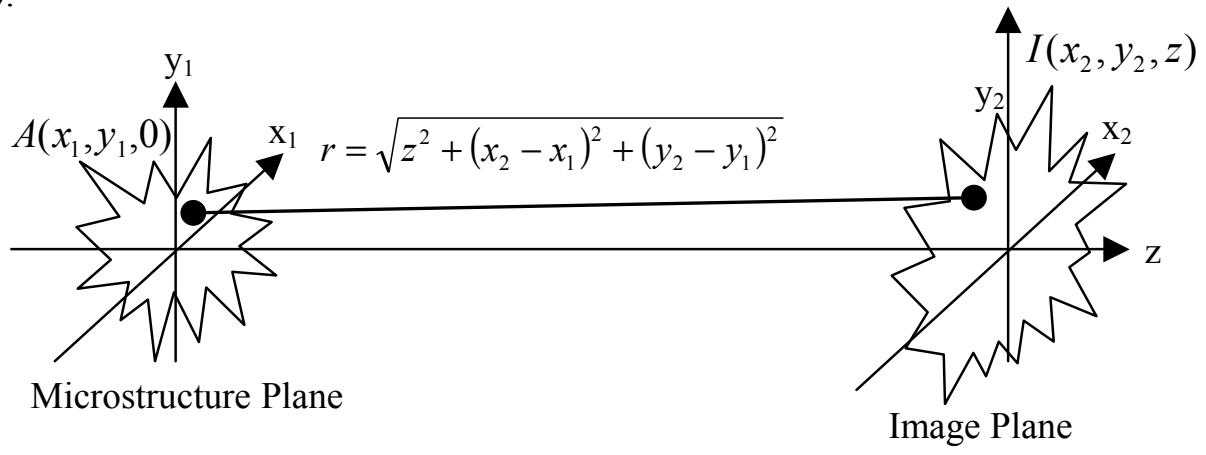

Figure 10 Coordinate system for computing diffraction

In the far field approach laid out in Fig. $9, I\left(x_{2}, y_{2}, z\right)$ is given by

$$
I\left(x_{2}, y_{2}, z\right) \propto\left|\mathfrak{I}\left\{A\left(x_{1}, y_{1}, 0\right)\right\}\right|^{2},
$$

where $\mathfrak{I}$ denotes the Fourier transform. The normalized intensity distribution of a micro-bead structure, which has circular apertures, yields a Bessel function of the first kind in the observation plan or airy diffraction pattern. The corner-cube material on the other hand has the diffraction pattern of a pentagon, which we can assume for the analysis to be a deformed square aperture function resulting in an intensity distribution $\sin ^{2}\left(\pi x_{2}\right) /\left(\pi x_{2}\right)^{2}$ in the one dimensional case. The theoretical intensity plot shown in Fig. 11 shows the micro-bead material producing larger side lobes, which generates less intensity in the main lobe, compared to a localized and more confined intensity from the corner-cube material. The amount of intensity in the main lobe is related to the level of brightness acquired in the image plane. Therefore, for an improved image quality, the phase conjugate material with a corner cube microstructure integrated in the HMD will provide better results in an imaging condition.
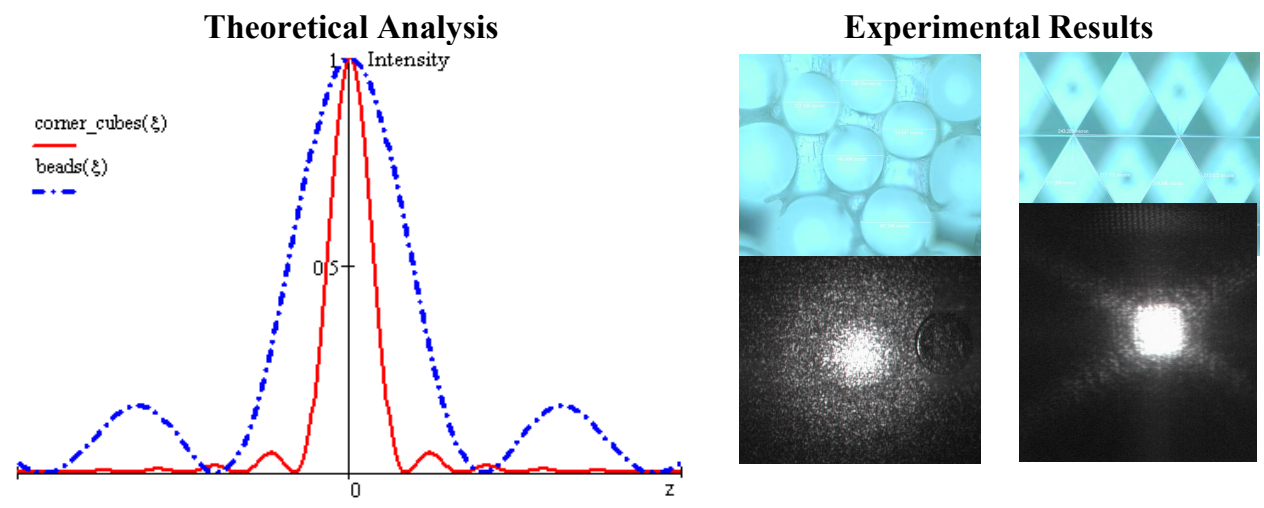

Figure 11 Intensity plot

\section{CONCLUSION}

The research presented here led to the design and the development of a novel HMD optical system consisting of a single unit assembly composed of a micro display, projection optics, and phase conjugate material all internally mounted inside the HMD. This unique design provides the capabilities of several applications such as, augmented reality for urban combat, guided surgery, 
and wearable computers allowing the user to view computer generated images in a see-through environment setting. This novel design also led to the assessment of two types of phase conjugate material that may be implemented in the ultra light weight head-mounted display assembly.

\section{ACKNOWLEDGMENTS}

We thank Rochester Photonic Corporation and Rick Plympton of Optimax Corporation for their generous assistance on the lens fabrication. We thank Vesselin Shaoulov and Yonggang Ha for stimulation discussion about this work. This research is supported by the US Army STRICOM as well as the Office of Naval Research grant number N000140310677.

\section{REFERENCES}

1. D. Buxton, G W. Fitzmaurice, "HMDs, caves and chameleon: a human-centric analysis of interaction in virtual space," Computer Graphics, Vol. 32, No. 4, 69-74 (ACM, 1998).

2. L. Davis, J.P. Rolland, F. Hamza-Lup, Y. Ha, J. Norfleet, and C. Imielinska, "Alice in the ARC: seamless transition between levels of immersion in virtual environments", IEEE Computer Graphics and Applications, 23(2), 10-12 (2003).

3. P.Milgram and F. Kishino, "A taxonomy of mixed reality visual displays," IECE Trans. Information and Systems (Special Issue on Networked Realidty), vol. E77-D, no. 12, 13211329 (1994).

4. F. Biocca and J.P. Rolland, "Teleportal face-to-face system," Patent Application Filed (2000).

5. R.F. Martins and J.P. Rolland, "Head-mounted display by integration of phase-conjugate material," Patent Application Filed (2003).

6. J. P. Rolland, V. Shaoulov, and F.J. Gonzalez, "The art of back-of-the-envelope paraxial raytracing", IEEE Transactions in Education (2001).

7. R. E. Fischer, "Optics for head-mounted displays," Information Display, vol.10, no.7-8, JulyAug. 12-16 (1994).

8. H. Hua, A. Girardot, C. Gao, and J. P. Rolland. "Engineering of head-mounted projective displays," Applied Optics, 39 (22), 3814-3824 (2000).

9. H. Hua, Y. Ha, and J.P. Rolland, "Design of an ultra-light and compact projection lens," Applied Optics 42(1),97-107, 2003. US patent filed (2002).

10. H. Ogawa, "Optical system with refracting and diffracting optical units, and optical instrument including the optical system," US patent 5,930,043 (1999).

11. J. B. Caldwell, "Diffractive apochromatic double-Gauss lens," Optics and Photonics News, 43-45 (Oct. 1999).

12. Y. Ha, and J.P. Rolland, "Optical assessment of head-mounted displays in Visual Space," Applied Optics 42(25), 5282-5289 (2002).

13. J.W. Goodman, "Introduction to Fourier optics," McGraw-Hill physical and quantum electronics series, New York, 1968. 